\title{
Antennas for multiple spot beam satellites
}

\author{
Michael Schneider - Christian Hartwanger • \\ Helmut Wolf
}

Received: 3 April 2011/Revised: 17 July 2011/Accepted: 18 July 2011/Published online: 10 August 2011

(C) The Author(s) 2011. This article is published with open access at Springerlink.com

\begin{abstract}
Ka-band payloads are becoming more and more popular for satellite communication. The wider band width in Ka-band allows a better satisfaction of the increasing demand for capacity. In addition to the use of more resources, a more efficient use of the available resources will become key for a successful development of satellite communication services. Modern antenna concepts allow a high frequency reuse scheme and, therefore, an extreme efficient use of the most rare resource in satellite communication, the frequency band width. In this paper, we describe the design and the use of different types of such antennas.
\end{abstract}

Keywords Multiple spot beam antennas .

Single feed per beam - Multiple feeds per beam

\section{Introduction}

In December 2010, the two first European Ka-band multi spot beam satellites, Avanti's Hylas- 1 and Eutelsat's Ka-Sat were launched. Both satellites were built by the European satellite manufacturer Astrium and are fully operational in orbit. Although most satellites in Ku-band provide large contoured coverages for broadcast applications, there is increasing interest in the use of spot beams for high-speed

This paper is based on a presentation at the German Aerospace Congress, September 27-29, 2011, Bremen, Germany.

M. Schneider $(\square) \cdot$ C. Hartwanger $\cdot$ H. Wolf

Astrium GmbH, 81663 Munich, Germany

e-mail: michael.schneider@astrium.eads.net broadband satellite services using Ka-band frequencies. Typical applications are personal communication, high speed Internet, military communication and mobile communication services. The service area has to be covered by many (up to 100) overlapping high gain spot beams to support two-way (uplink and downlink) broadband services using small terminals. This approach allows a high degree of frequency reuse, which leads to enormous increase of system capacity. A multiple spot beam satellite for an European coverage can provide a more than ten times higher capacity than classical satellites using the same input power and similar antenna dimensions.

For the creation of a multiple spot beam scenario with overlapping spots, the antenna system is a key component. Two basic principles are possible. Single feed per beam (SFB) designs use one feed horn for each spot. Advantages are hardware simplicity and a slightly better electrical performance, but at the expense of an increased number of apertures. To provide overlapping spots, in this case several reflector apertures, typically four, are required. Often, it is also possible to create a four colour scenario using only three reflectors. Additionally designs using a single oversize shaped reflector [1], passive [2] or active [3] lenses are possible as well. Multiple feeds per beam (MFB) designs use small sub-arrays for each spot. Adjacent spots share some of the array elements. In this case, overlapping feed arrays are created, which allow producing overlapping spots using a single reflector aperture. The elements of the array are fed by a complex orthogonal beam forming network.

In the paper, we discuss both principles, present designs and compare simulated and measured performance data. Antennas with up to 100 individual overlapping spots also require a new test philosophy. Therefore, we also present equipment and new efficient test methods for multiple spot beam antennas. 


\section{Multiple spot beam scenarios}

To achieve a high degree of frequency reuse, the target coverage is not longer covered by a large single beam, but by a high number of overlapping high gain spot beams. Figure 1 shows this principle for a pan-European coverage, where a four colour frequency reuse scheme is used. This means two different frequency sub-band and two orthogonal polarisations (usually right hand and left hand circular) are used. Spots with different colours differ in frequency or polarisation. Therefore, they can transfer different information without mutual interferences. Spots with the same colour use the same frequency and the same polarisation, but they are spatially isolated from each other, no spot has a neighbour with the same colour. In this way also, spot beams with the same colour can transfer different information. In most cases, the four colour scenario is the best compromise between system capacity and performance. However, also other frequency re-use schemes, for example, a three or a seven colour scheme can be used [4].

The frequency reuse factor of a multi-spot beam antenna, as compared to a standard large contour beam antenna, is the number of spots divided by the number of colours. For example, an European four colour scenario with 80 spot beams has a frequency reuse factor of 20 . Because of the overlap of the beams and other technical constrains this factor reduces to about 12 . However, this means a multi-spot beam satellite with 80 spot can provide about 12 times more capacity than a satellite with a large contour beam and therefore a 12 times higher sales potential can be achieved. This exceptional increase in satellite capacity is achieved without an increase in RF or DC power, without an increase in mass and only minor increase in cost for the satellite.

More and more communication satellites use Ka-band. The Ka transmit (Tx) band (from satellite point of view or

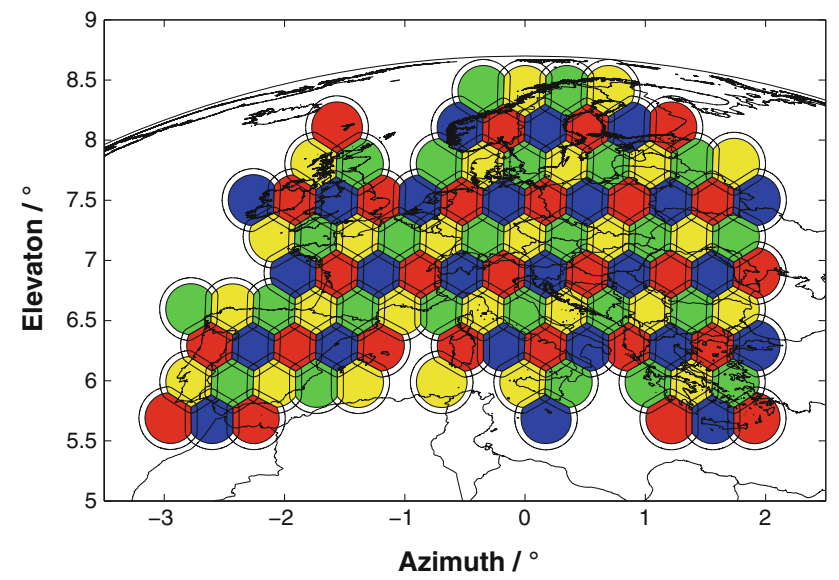

Fig. 1 Pan-European multi spot beam coverage downlink band) is around $20 \mathrm{GHz}$ the receive ( $\mathrm{Rx}$ ) band (uplink band) around $30 \mathrm{GHz}$. Modern Ka-band multi-spot beam satellites will be able to provide broadband services to areas with an underdeveloped terrestrial infrastructure.

\section{Single feed per beam antennas}

Single feed per beam antennas are the current state of the art for multiple spot beam antennas. Each beam is created by a single horn. To avoid holes in the illumination of the coverage, overlapping spot beams are required. This can be achieved using a single oversize shaped reflector [1], passive [2] or active [3] lenses. The currently most popular principle is to create a virtual overlapping using four standard unshaped reflector antennas, one for each colour [4]. Figure 2 demonstrates the principle. Each reflector aperture creates non-overlapping spots of a single colour. The four antennas are pointed such that a four colour scenario with overlapping spots is achieved. Strictly spoken eight reflectors would be required, four for transmit (Tx) and four for receive (Rx). This can be avoided if complex Tx/Rx feed chains are used. Because the reflector diameter is chosen for the transmit frequency, it is too large for the receive frequencies. The spots would become too small and not overlap any longer. Therefore, a clever feed horn design is required to provide a proper illumination of the reflector at $\mathrm{Tx}$ frequencies and a specific under-illumination in the receive band.

Figure 3 shows the engineering model of a combined Tx/Rx user and gateway feed chain. This feed chain was developed at Astrium $\mathrm{GmbH}$ in the frame of an ESA Artes 5.2 contract. In the frame of an ESA Artes 3-4 contract the development and qualification of an engineering qualification model is currently running. The feed chain consists of several milled layers that contain a diplexing turnstile, magic-Ts, a branch-line coupler and a septum polariser.

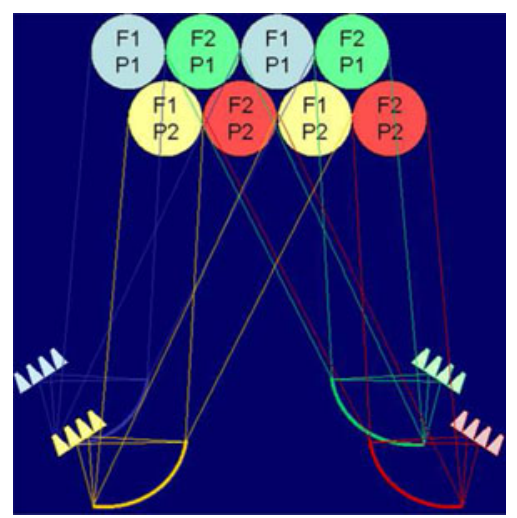

Fig. 2 A four colour scheme using four reflectors 


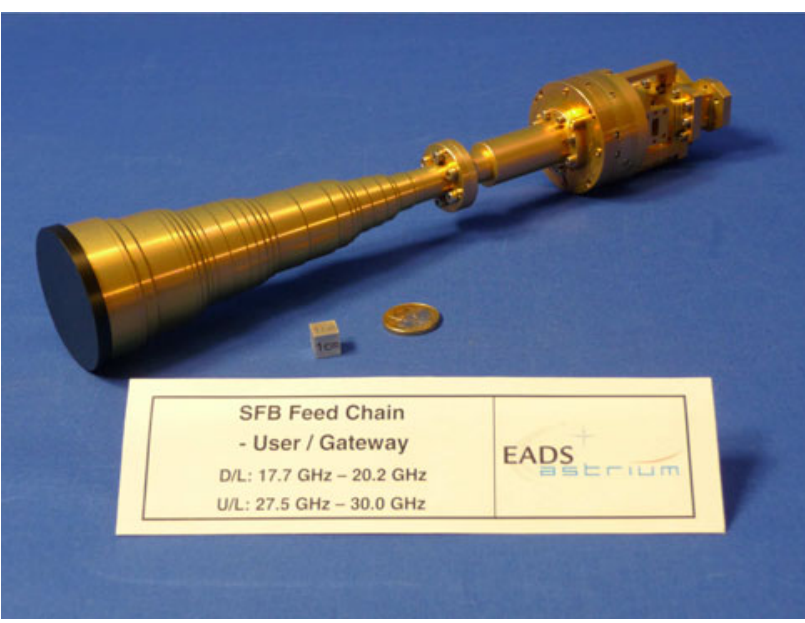

Fig. 3 Engineering model of a user gateway feed chain

The measured return loss of the feed chain is shown in Fig. 4. More measurement results were published in [5].

Multi-spot beam antennas provide beams with a typical diameter of $0.5^{\circ}$ or less. Therefore, a very accurate pointing of the antenna is required. This can for example be achieved by the use of an active pointing system. A beacon signal is received by a special RF sensing feed chain, which can provide both, a sum and a delta pattern. From amplitude and phase of this tracking signal, an error vector for an automatic pointing adjustment can be derived. One RF sensing feed chain for each reflector is required. Often the beacon station is located inside the user area. Therefore, the RF sensing capability has to be provided by the feed chain additionally to the user and gateway function. Figure 5 shows an engineering model of a combined $\mathrm{Tx} / \mathrm{Rx}$ user, gateway and RF sensing feed chain. This feed chain was developed at Astrium $\mathrm{GmbH}$ in the frame of an ESA Artes 5.2 contract [6]. The development and qualification of an engineering qualification model is currently running in the frame of an ESA Artes 3-4 contract. Figure 6 shows the measured sum and delta pattern of the RF sensing feed chain.

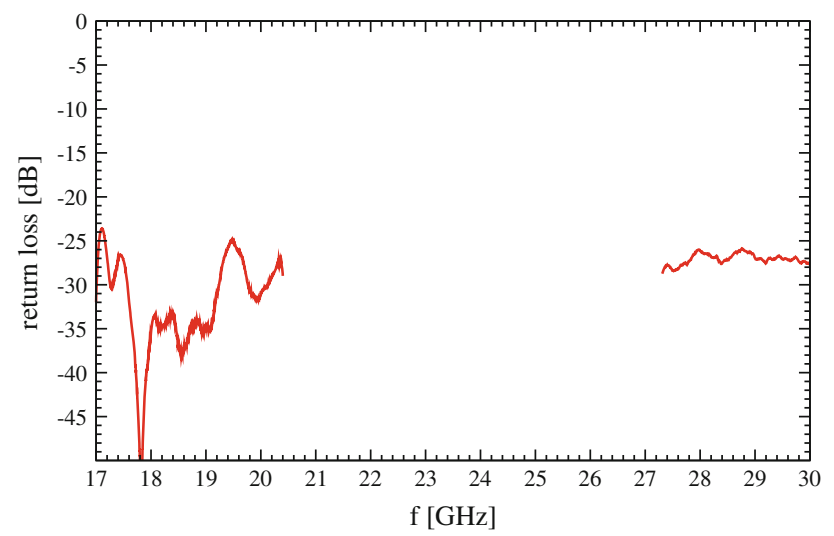

Fig. 4 Measured return loss user gateway feed chain

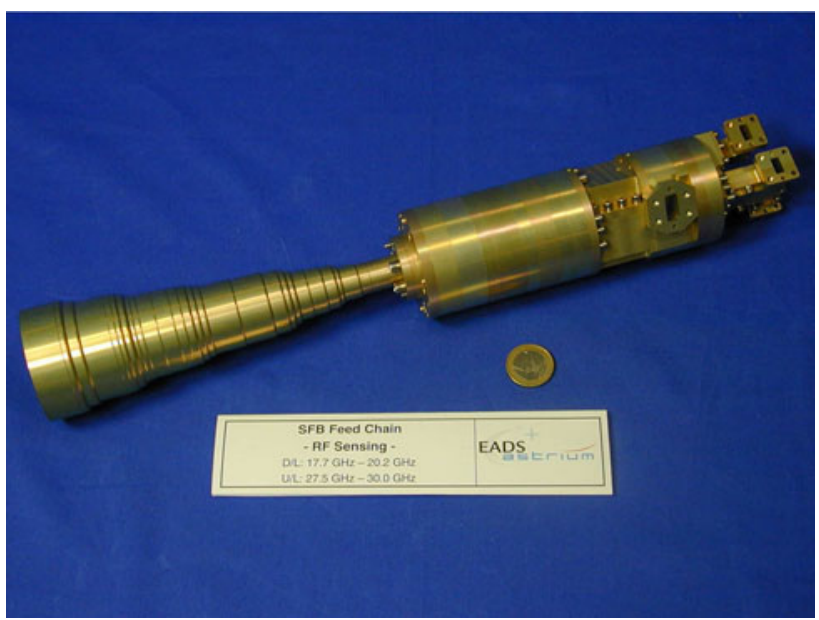

Fig. 5 Engineering model of an RF sensing feed chain

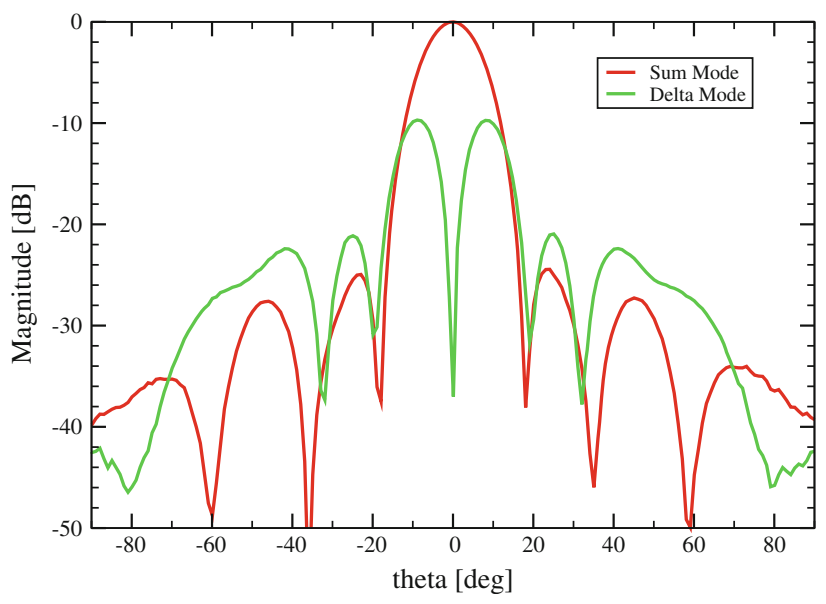

Fig. 6 Measured sum and delta pattern of the developed RF sensing feed chain

\section{Multiple feeds per beam antennas}

Figure 7 shows the engineering model of a multiple feeds per beam feed system, which was developed at Astrium $\mathrm{GmbH}$ in the frame of the DLR granted project "Medusa" [7]. The development and qualification of an engineering and qualification model is currently running in the frame of an ESA Artes 3-4 program. MFB antennas create the beams using arrays of small horns. Adjacent beams share horns (Fig. 8). Owing to this physical overlap of the feed apertures, overlapping spots can be produced using only one reflector aperture. This leads to a significant reduction in mass and cost and simplifies the accommodation of the antennas on the space craft [8]. These advantages are achieved at the expense of a complicated beam forming network (BFN). Figure 9 shows the partly assembled Medusa BFN. For larger coverages, several hundreds of 


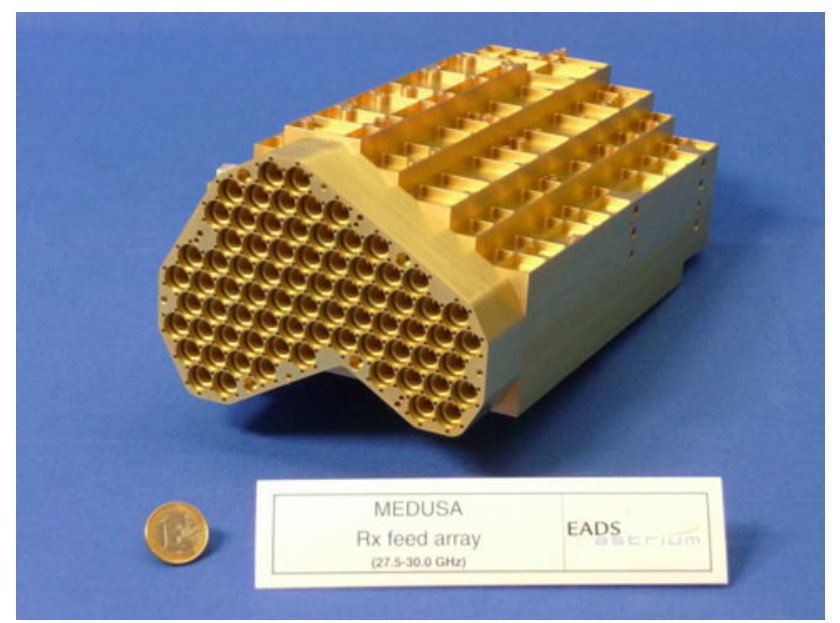

Fig. 7 Engineering model of the Medusa feed system

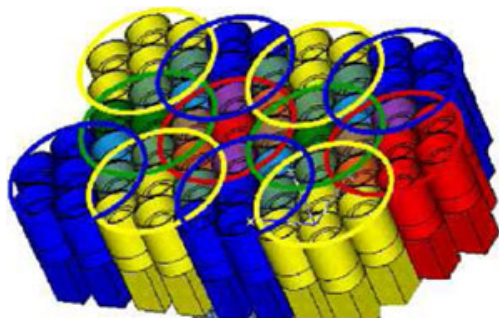

Fig. 8 MFB feed system with shared horns

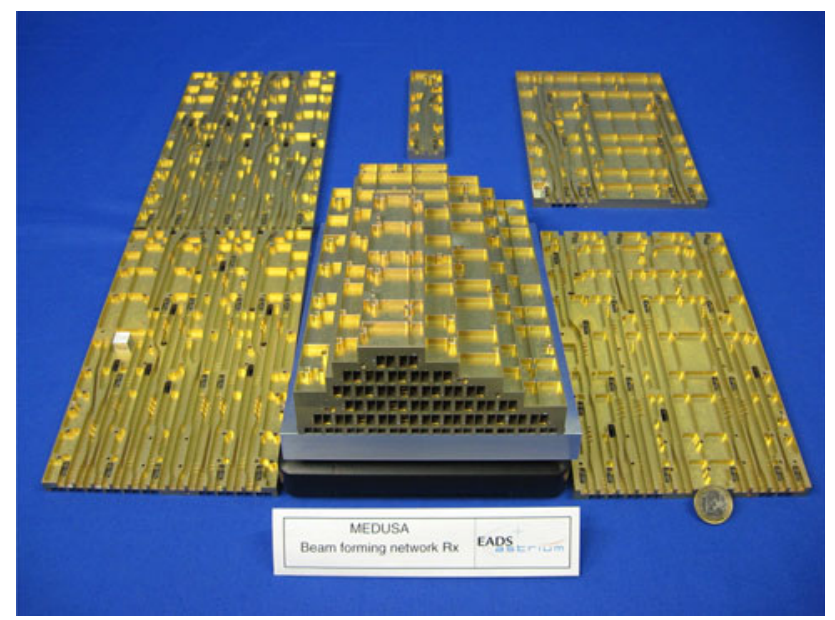

Fig. 9 Partly assembled BFN of the Medusa feed system

couplers and phase shifters have to be designed and accommodated in the different layers of the BFN. Advanced design methods were developed to design, such BFNs [9].

Because separated antennas for transmit and receive are used, the reflector diameter can be sized optimally for frequency and spot diameter.

\section{Comparison of SFB and MFB concept}

Single feed per beam and multiple feeds per beam are complementary concepts. There is no strict assignment of the concepts to different scenarios. However, a few guidelines for the selection shall be given in this section.

SFB antennas have a slightly better gain performance than MFB antennas. Therefore for large scenarios, like the one in Fig. 1, on large spacecrafts (e.g. E3000), SFB antennas should be advantageous. However, for very large scenarios, the scan losses can become quite high. In such cases, it should be discussed whether the four SFB antennas can be replaced by four MFB antennas, two for Tx and two for Rx. In this case, each MFB antenna has to provide only a half of the coverage and so the scan losses could be significantly be reduced. The cost and mass for both concept are comparable.

Main advantage of the MFB concept is the need of only two reflectors, one for Tx and one for Rx. On large space crafts both antennas can be accommodated on the same side panel. The second side panel could be used for C- or Ku-band antennas. When compared with a pure Ka-band satellite in this way the effects of market fluctuations can be reduced.

Smaller satellite buses are widely used. In general, it is difficult to accommodate four large reflectors on these buses. In addition, the available RF power is only sufficient for a relative small number of beams. The use of four large reflectors for a small number of beams is hardly profitable. Therefore, for smaller space crafts and smaller scenarios, as the central European scenario shown in Fig. 10, MFB antennas should be the preferred choice.

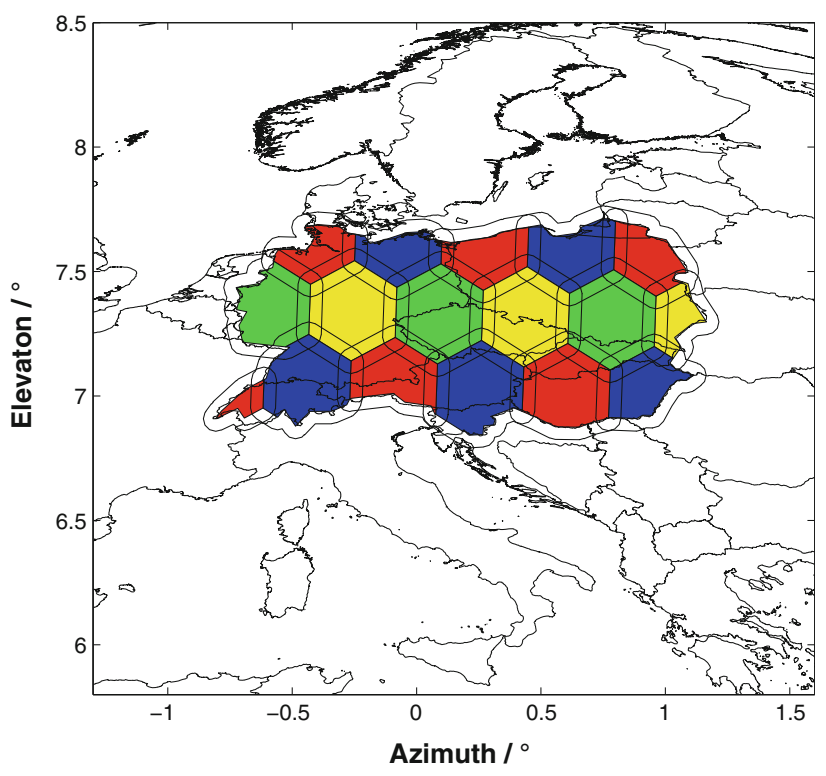

Fig. 10 Central European spot beam scenario 


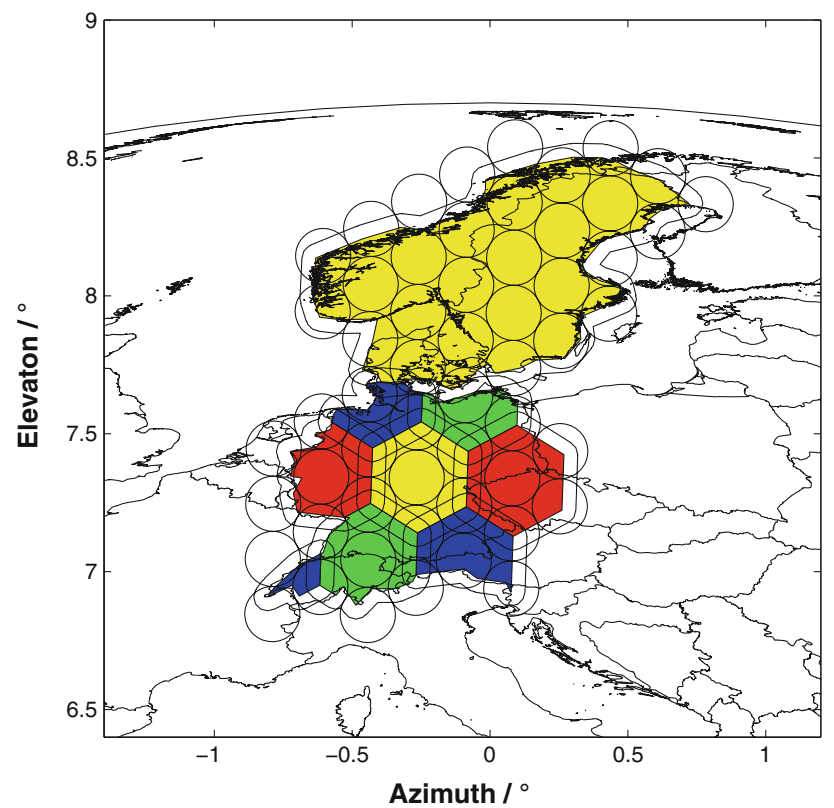

Fig. 11 Spot size can be adapted to capacity needs

C-band satellites are still important to serve existing customers and for areas with high rain attenuation. As the available bandwidth in C-band is relatively low, frequency reuse can be very important. Because of the low frequency, a SFB solution could become quite heavy and bulky. A MFB antenna, with a barline BFN instead of a waveguide BFN, would be a more promising concept.

Mid- and long-term a flexible power to beam allocation, depending on the required capacity of the spot, will be realised using matrix power amplifiers [10] and switch matrices [11]. Short-term, until space qualified flexible Kaband payload becomes available, different spot diameters can be chosen to account for different capacity needs. An example is shown in Fig. 11. The antenna illuminates Germany, Switzerland and Austria, areas with a highpopulation density, with small spots, formed by a standard configuration with seven horns per beam. The same antenna illuminates the Scandinavian countries, areas with a lower population density, with a single large spot, formed by a higher number of horns. In this way, a better balancing of the capacity becomes possible.

\section{Multiple contour beam scenarios}

Multifeed antennas can also be used for contour beams. Especially for smaller linguistic beams for modern broadcast services an increasing need is likely. Figure 12 shows a typical linguistic beam concept. The level of frequency reuse is lower than for spot beams, but at least it is possible to increase the satellite capacity. For most TV stations, it is

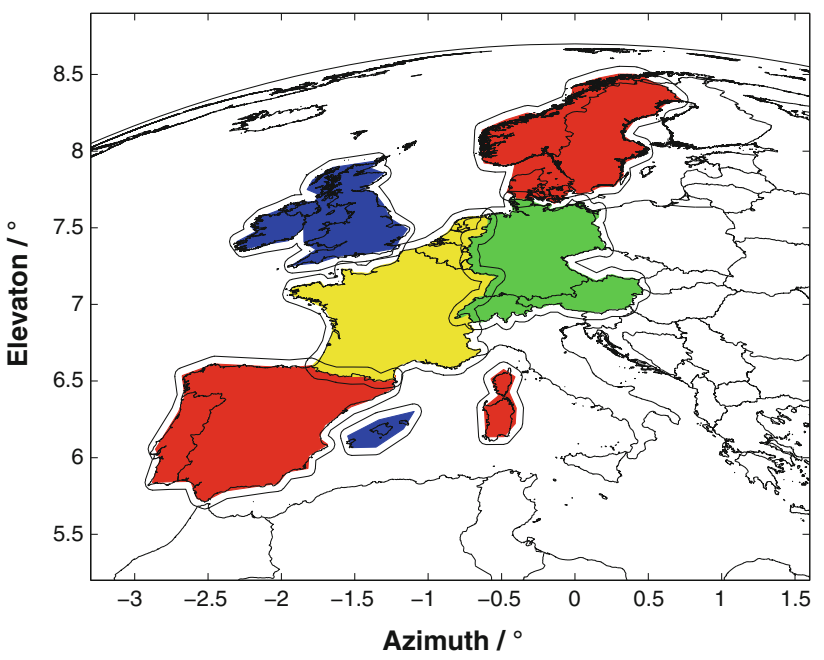

Fig. 12 European language beam scenario

sensible or even advantageous (license fee for sport events or movies), if the reception is limited to a certain political or linguistic area. The beams can be sized for single countries, groups of countries, islands etc. A high isolation between areas with the same colour is required. If the distance between contour beams using the same colour is low, a large reflector is required to achieve a high isolation. An advantage of multifeed antennas is the high degree of freedom for the excitation coefficients. This makes it easier to suppress side lobes for a high isolation between the different coverages.

\section{RF measurements of multi spot beam antennas}

The accuracy of the excitation coefficient is essential for the performance of MFB antennas. Therefore, it is necessary to measure them already during the assembly of the BFN using a network analyser. Figure 13 shows the measurement set-up

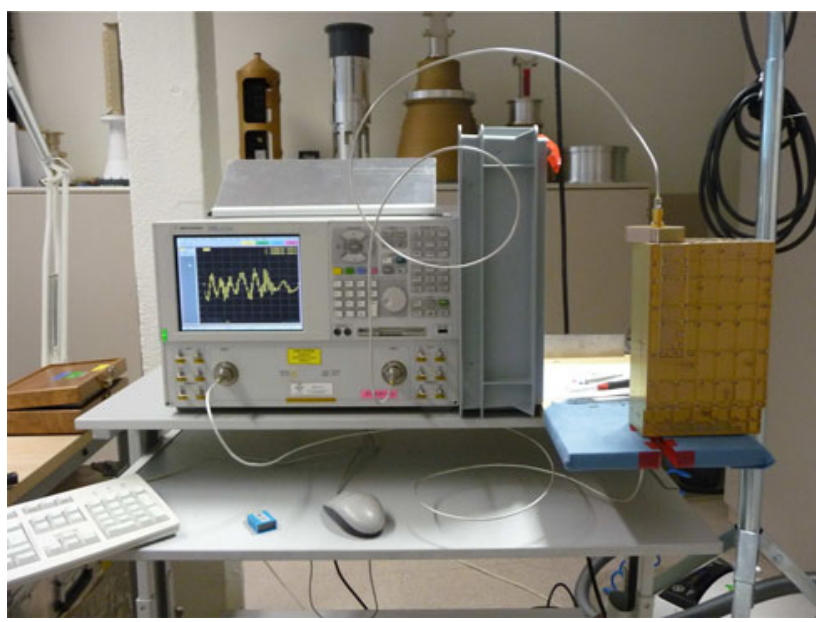

Fig. 13 S-parameter measurement of the BFN 
in a clean room at Astrium GmbH. The BFN has one input port for each beam and typically seven output ports, so seven sets of S-parameters have to be measured for each beam. For typical multi-spot beam scenarios a few hundreds S-parameters have to be measured. Although one output port is measured all other output ports that belongs to the same beam must be terminated by a matched load. Special measurement adaptors are required for this task. As the calibration for the phase measurement is sensitive against temperature changes, the temperature has to be kept constant during the measurement. These measurements allow in verifying the accuracy of the manufacturing process and a prediction of the antenna performance. For a transmit feed also the dissipation power, which is caused by ohmic losses, can be determined.

Figure 14 shows a comparison between measured and predicted magnitudes of the excitation coefficients for one beam of the Medusa BFN and Fig. 15 a comparison of the phases. A very good agreement between measurement and prediction for both, amplitudes and phases was achieved. This good agreement is an evidence for the maturity and precision of the design process and the manufacturing of the beam forming network.

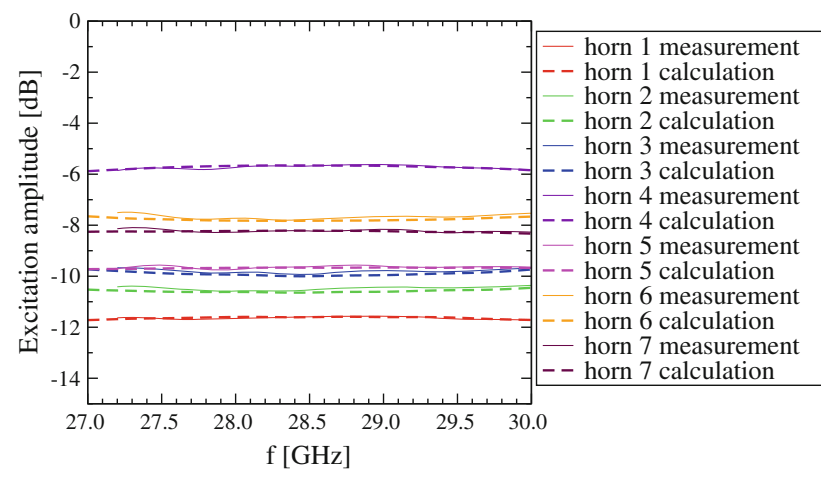

Fig. 14 Comparison between measured and calculated magnitudes of the excitation coefficients of one beam

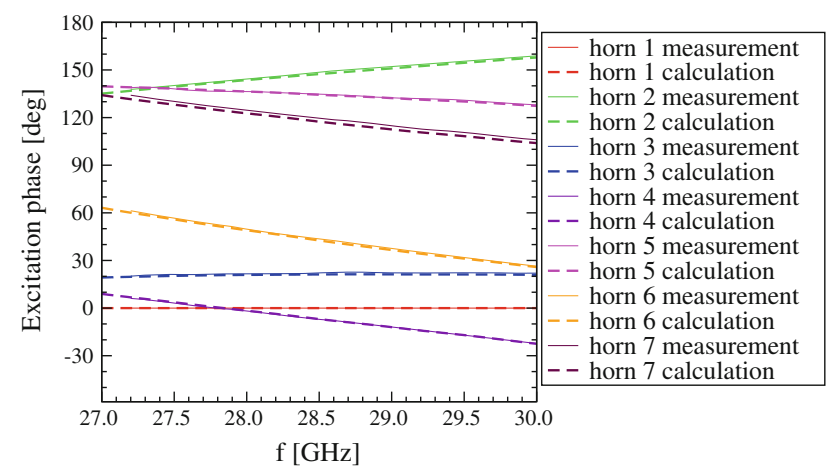

Fig. 15 Comparison between measured and calculated phase of the excitation coefficients of one beam

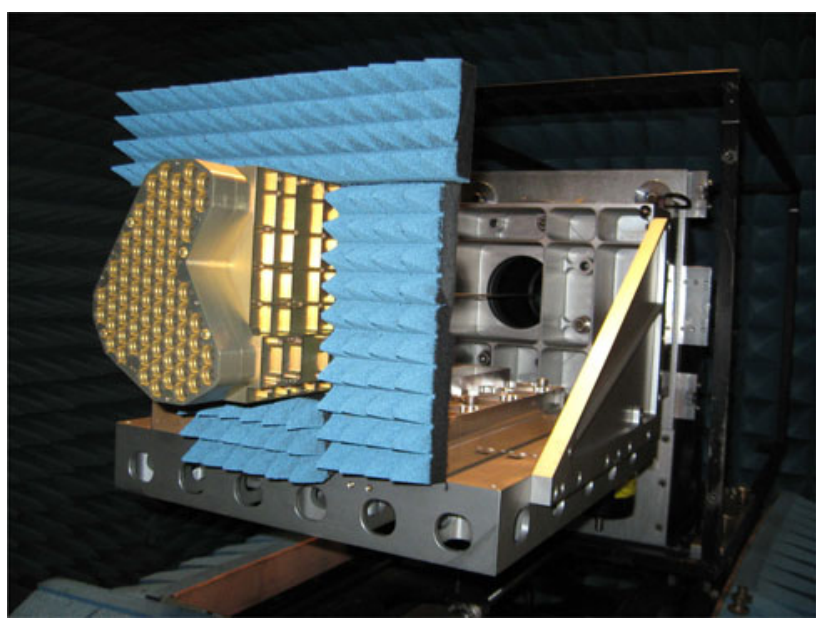

Fig. 16 Feed pattern measurement in a feed test range

Before the measurement of the entire antenna, the feed system should be measured on its own. Figure 16 shows the pattern measurement of the Medusa feed system in the feed test range of the Astrium GmbH. The co- and cross-polar pattern and the gain for each beam has to be measured.

Pattern measurements for satellite antennas are usually performed in compensated compact ranges (CCR). CCRs can provide far field conditions inside a chamber with controlled environmental conditions. The dual reflector principle allows measurements with very low cross-polar levels and excellent accuracy [12]. Figure 17 shows the Medusa antenna during the measurement in the compensated compact range of the Astrium GmbH [13]. The spots of a multi-spot beam antenna have a very narrow beam width. Typical half-power beam widths are between 1.0 and $0.2^{\circ}$. Therefore, the measurements have to be performed using a very dense angular resolution. All beams have to be measured across the entire service area, because the crosspolar and side lobes are interferer for other beams. The effort for the measurement of each beam is comparable to the effort for a standard antenna. Therefore, new measurement procedures were developed, which allow a quasiparallel measurement of a high number of beams [14].

Figure 18 shows the measured pattern of the Medusa antenna, which was designed to provide spot beams for the Arabian peninsula, Syria, Egypt and a part of Libya. In addition, the alignment of the feed system against the reflector and the reflector with respect to the coordinate system of the compact range is of high importance. The traditionally alignment using theodolites was replaced by a modern laser tracking-based system. A laser tracker measures the positions of retro target on the feed system, the antenna reflector and the reflectors of the compact range. From the measurements, correction vectors for the positioners are derived. The positioners correct the position of feed system and antenna until the correction vectors keep 


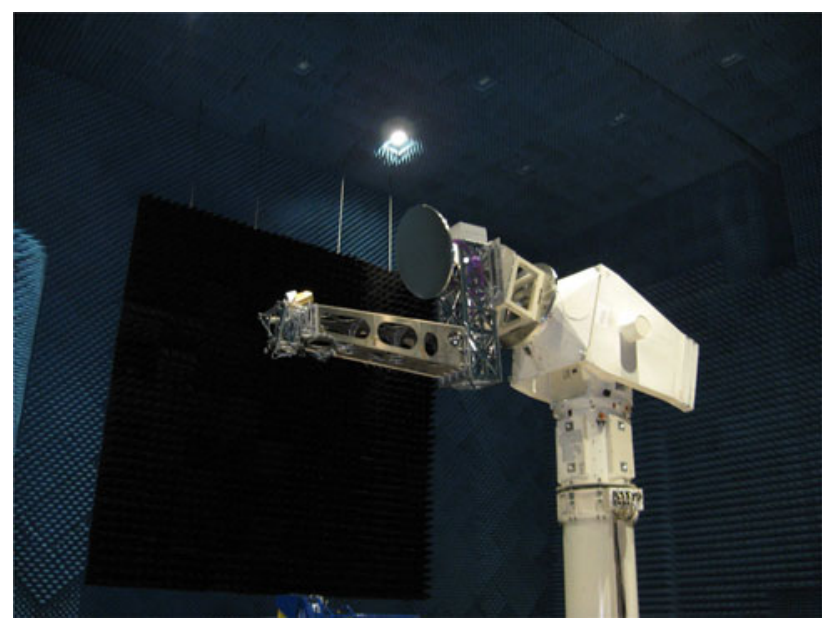

Fig. 17 Measurement of the antenna pattern in a compensated compact range

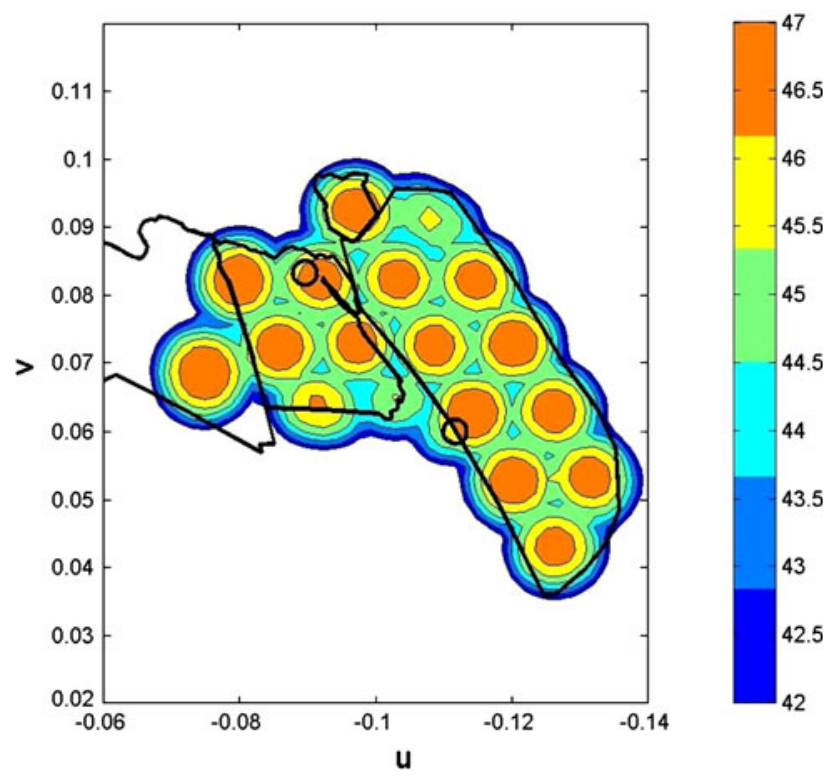

Fig. 18 Measured directivity of the Medusa antenna

below a specified threshold. Figure 19 shows the laser tracker and the positioner for the feed system during the alignment in the compact range of the Astrium GmbH.

\section{Conclusion and outlook}

Multiple spot beam satellites can provide a much higher capacity than conventional satellites. The massive increase in capacity is mainly achieved by application of a high degree frequency reuse scheme. The much more efficient use of the available resources will allow satellite providers to offer competitive services for broadband and broadcast. Multi-spot beam antennas are a key element for the frequency reuse scheme. The two basic concepts, single feed

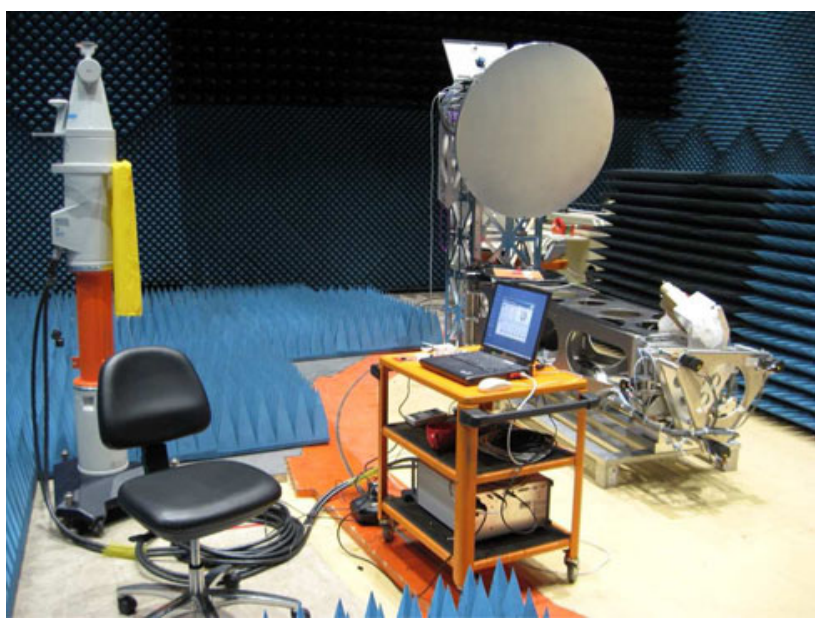

Fig. 19 Alignment of the Medusa feed system using laser tracker and positioner

per beam and multiple feeds per beam, and their application were discussed. Multi feed antennas can be use on large satellite platforms, as well as on smaller platforms. Multi-spot beam concepts are promising for both, upcoming frequency bands like Ka-band and traditional frequency bands like C- and Ku-band.

Current payloads for multi-spot beam satellites have a limited flexibility. The German Space Agency supports a wide range of developments for future flexible payloads, for example switch matrices [11], flexible power amplifiers [15], flexible liquid crystal phase shifters [16], flexible beam forming networks [17], etc. These technologies will allow in integrating reconfigurability and flexibility into the existing multi-spot beam concepts.

Acknowledgments Our work for the development of multiple spot beam antennas has been supported by the German Federal Ministry of Economics and Technology (BMWi) under the project management of the German Space Agency (DLR, Grant 50YB0714) and by the European Space Agency (ESA, Contracts 400010915/10/NL/US, 22817/09/NL/US). The authors like to thank DLR and ESA for support and fruitful discussion.

Open Access This article is distributed under the terms of the Creative Commons Attribution Noncommercial License which permits any noncommercial use, distribution, and reproduction in any medium, provided the original author(s) and source are credited.

\section{References}

1. Balling, P., Mangenot, C., Roederer, A.G.: Shaped single-feedper-beam multibeam reflector antenna. In: First European Conference on Antennas and Propagation (EuCAP) (2006)

2. Reiche, E., Gehring, R., Schneider, M., Hartwanger, Ch., Hong, U., Ratkorn, N., Wolf, H.: Space fed arrays for overlapping feed apertures. In: German Microwave Conference (GeMIC) (2008) 
3. Ruggerini, G., Toso, G., Angeletti, P.: An aperiodic active lens for multibeam satellite applications: from the design to the breadboard manufacturing and testing. In: Fifth European Conference on Antennas and Propagation (EuCAP) (2011)

4. Rao, S.K.: Parametric design and analysis of multiple-beam reflector antennas for satellite communications. IEEE Antennas Propag. Mag. 45, 26-34 (2003)

5. Hong, U., Stirland, S., Gehring, R., Hartwanger, C., Wolf, H.: A Dual Circular Combined K/Ka-Band User and Gateway Feed Chain for Multi Beam Satellite Antennas. 32nd ESA Antenna Workshop, Noordwijk (2010)

6. Reiche, E., Stirland, S., Hartwanger, C., Hong, U., Gehring, R., Wolf, H.: A dual circular combined K/Ka-band RF sensing feed chain for multi beam satellite antennas. In: Fifth European Conference on Antennas and Propagation (EuCAP) (2011)

7. Ratkorn, N., Schneider, M., Gehring, R., Wolf, H.: MEDUSAA Multiple Feeds Per Beam Multi Spot Beam Antenna Project, pp. 59-62. 30th ESA Antenna Workshop, Noordwijk (2008)

8. Schneider, M., Hartwanger, C., Sommer, E., Wolf, H.: The multiple spot beam antenna project medusa. In: Third European Conference on Antennas and Propagation (EuCAP), pp. 726-729 (2009)

9. Reiche, E., Schneider, M., Gehring, R., Wolf, H.: Efficient Antenna Design Using 3D EM and Circuit Co-simulation. 54th International Scientific Colloquium, Ilmenau (2009)

10. Couchman, A., Jones, D.: Optimized multiport amplifiers for wideband multi-beam satellites. In: 24th AIAA International
Communications Satellite Systems Conference (ICSSC), San Diego (2006)

11. Humbla, S., Müller, J., Stephan, R., Stopel, D., Trabert, J.F., Vogt, G., Hein, M.A.: Reconfigurable Ka-band switch matrix for on-orbit verification. In: European Microwave Conference, pp. 610-613 (2009)

12. Dudok, E., Fasold, D., Steiner, H.-J.: A new advanced test centre for communication satellite antenna and payload testing. In: First European Conference on Satellite Communications (ECSC), pp. 183-196 (1989)

13. Schneider, M., Hartwanger, C., Sommer, E., Wolf, H.: Test results for the multiple spot beam antenna project medusa. In: Fourth European Conference on Antennas and Propagation (EuCAP) (2010)

14. Hartmann, J., Habersack, J., Steiner, H.-J.: Improvement of Efficiency for Antenna and Payload Testing. 30th ESA Antenna Workshop, Noordwijk (2008)

15. Voigt, S.: TWT's in space-present status and future applications. IEEE Int. Vacuum Electr. Conf. 53-56 (2009)

16. Gäbler, A., Gölden, F., Manabe, A., Göbel, M., Müller, S., Jakoby, R.: Investigation of high performance transmission line phase shifters based on liquid crystal. In: European Microwave Conference (2009)

17. Uhlig, P., Leiss, J.; Marek, R.; Sommer, J.-P.; Wolf, H.: Light weight-low loss beam forming networks for space applications. In: Third European Conference on Antennas and Propagation, pp.740-744 (2009) 